\title{
On citing well
}

\section{Scientists need to devote more attention to the citation lists of scientific papers-the connectivity and usefulness of the scientific literature depend upon it.}

The appearance of new ideas and discoveries in the scientific literature is a reflection of ongoing scientific progress. Individual articles are nodes of scientific knowledge, but citations of published work link together the concepts, technologies and advances that define scientific disciplines. Though information technology and databases have helped us to better manage the expanding scientific literature, the quality of our citation maps still hinges on the quality of the bibliographic information contained in each published paper. Because article citations are increasingly used as metrics of researcher productivity, the citation record also affects individual scientists and their institutions. As a result, all participants in the scientific publication process need to ensure that the citation network of the scientific literature is as complete and accurate as possible.

Many factors may stand in the way of good citation practices. As scientific papers are written, the emphasis on the data and the interpretation of results may overshadow other aspects of manuscript preparation, including the construction of a complete and coherent reference list. Each research group has its own referencing habits, and some may feature their own work too prominently or rely on familiar references without a critical examination of whether a particular citation is the most appropriate in the given context. Some researchers may not cite 'old' papers either because these are incorrectly viewed as being out of date or because inertia inevitably may encourage authors to cite the articles that show up more frequently in searches or that have appeared recently. Finally, more prominent citation of review articles, instead of original research papers, can obscure or bias the connectivity of the scientific literature (Nat. Cell Biol. 11, 1, 2009). Though review articles are appropriately used as overview citations for broad scientific topics or ideas, most citations, especially those focusing on previously published concepts or results, should be of original research papers.

Researchers understandably are motivated, in both professional and personal ways, to have their scientific contributions recognized through citation by their peers. The community also values the accurate assignment of credit and precedence for scientific discoveries. As a result, even an accidental omission of a necessary citation may create an uncomfortable situation for a paper's authors. More problematic, however, are cases where authors deliberately omit relevant citations. Because perceived novelty can be an important factor in determining where a manuscript is published, some authors may be tempted to avoid citing earlier or concurrent work from their own laboratories to enhance the apparent advance of a submitted study. In other cases, some authors may consider ongoing scientific disagreements, personal conflicts or competition a sufficient justification for omitting citations of work by others. Clearly authors need to do everything they can to avoid accidental omission of key references, and should never exclude relevant citations for nonscientific reasons. In turn, all scientists, independent of their roles as authors, referees or editors, need to renew their commitment to guaranteeing that literature citations correctly assign credit for ideas and discoveries and are placed thoughtfully in manuscripts and published papers.

At Nature Chemical Biology, we are committed to ensuring that the reference lists of our published papers are accurate and balanced. As part of our assessment of new manuscripts, we look closely at the study in the context of published literature, which includes the paper's citation list. We also require that authors include confidential copies of related manuscripts submitted elsewhere (see "Submission Policies" at http://www.nature.com/ nchembio/authors/submit/index.html) for consideration by editors and referees during manuscript assessment. During the review process, we explicitly ask referees to comment on the work in the context of the current literature. We also enforce reference limits-currently 50 references for research articles and 25 for brief communications (increased from 15 in the fall of 2008) - because we believe that these limits reinforce thoughtful reference selection by authors. Finally, because we understand the fast-paced nature of chemical biology research, we do our best to coordinate publication of competing manuscripts with other journals. In cases where that is not possible and a Nature Chemical Biology paper appears after a related publication elsewhere, we expect that authors will cite the related study appropriately in their revised manuscript.

Though editors and referees can help, authors are ultimately responsible for the information contained in their published papers. We recommend that authors take several important steps to increase the quality of their citation lists. First, principal investigators need to teach young scientists the appropriate ways to select manuscript references and mentor them in the ethical dimensions of citation. Second, authors need to put as much care into selecting and accurately citing references as they devote to the rest of their manuscripts. As part of this process, authors should perform comprehensive literature searches as they write and revise manuscripts, so as to identify relevant work that may need to be cited. Before including references in their citation lists, all authors should have read and discussed the candidate references to ensure that they are the most relevant choices and are called out at the appropriate point in the paper. Finally, authors need to be more transparent in informing editors and referees of related work that is being considered for publication in parallel with submitted work.

The responsibility for maintaining and enhancing the citation network of a discipline resides with all participants: authors, referees, editors and database managers. Thoughtful attention during the writing and review processes remains the first and best approach for ensuring citation quality and the appropriate assignment of credit in published papers. Yet new publishing and database tools that lead us to an interactive multidimensional scientific literature will become essential. As publishers move toward integrating functionality such as real-time commenting on published papers and creating 'living manuscripts' that preserve the snapshot of a research area through the lens of a published paper, while permitting forward and backward linking, the scientific literature is poised to become a richer environment that will support future scientific progress. 